PRINCIPIOS DE POLÍTICA SOCIAL Y ECONÓMICA. ECONOMÍA Y HACIENDA 
: 


\title{
13. LOS DERECHOS SOCIALES \\ $Y$ LOS PRINCIPIOS RECTORES \\ DE LA POLÍTICA SOCIAL Y ECONÓMICA
}

\author{
SANTIAGO SÁNCHEZ GONZÁLEZ \\ Profesor Titular de Derecho Constitucional \\ UNED \\ $y$ \\ ANTONIO-CARLOS PEREIRA MENAUT \\ Profesor Titular de Derecho Constitucional \\ Universidad de Santiago de Compostela
}


SUMARIO

INTRODUCCIÓN.-I. IMPORTANCIA DEL PROBLEMA.-II. Algunas ACLARACIONES.-III. Statu quo.-IV. Opciones.-V. Nuevo articulado. 


\title{
13. LOS DERECHOS SOCIALES Y LOS PRINCIPIOS RECTORES DE LA POLIITICA SOCIAL Y ECONÓMICA
}

\author{
POR \\ SANTIAGO SÁNCHEZ GONZÁLEZ \\ Profesor Titular de Derecho Constitucional \\ UNED \\ y \\ ANTONIO-CARLOS PEREIRA MENAUT \\ Profesor Titular de Derecho Constitucional \\ Universidad de Santiago de Compostela
}

INTRODUCCIÓN económica.

Los derechos sociales y los principios rectores de la política social y

I. El día 18 de febrero de 1992, el diario El País publicaba una breve noticia anunciando que «EI Ministerio de Industria intenta que la multinacional Du Pont de Nemours amplíe sus inversiones en Asturias. En concreto, en investigación (tecnológicas, laboratorios o plantas pilotos)".

El 9 de marzo, otra breve noticia en el mismo diario informa que "Du Pont no quiere a los sindicatos en su nueva factoría asturiana", es decir, que «no contratará a trabajadores afiliados a los sindicatos". 
El 10 de marzo, el mismo periódico informa que "CC.OO. y UGT solicitaron ayer públicamente que el Gobierno retire las subvenciones concedidas a Du Pont por no querer contratar a sindicalistas".

II. Por otro lado, la Constitución española de 1978 es una de las pocas que, por osadía de sus redactores, recoge en el art. 45 uel derecho de todos a disfrutar de un medio ambiente adecuado" y obliga a "los poderes públicos a que velen por la utilización racional de todos los recursos naturales, con el fin de proteger y mejorar la calidad de vida y defender y restaurar el medio ambiente". Además, dispone que "se establecerán sanciones penales o administrativas para quienes violen lo estipulado en el anterior apartado", con la obligación adicional de "reparar el daño causado".

En ejecución del mandato constitucional, por fin, en el año 1983, se tipificó el delito contra el medio ambiente en el artículo 347 bis del Código Penal, previendo penas de arresto mayor y multa de hasta cinco millones de pesetas e, incluso, la clausura temporal o definitiva del establecimiento causante.

También se han promulgado diversas normas de rango y alcance variado para proteger las aguas, las especies animales, espacios naturales, etc.

III. La empresa multinacional norteamericana Du Pont de Nemours no representa dignamente la figura del fisiócrata cuyo nombre adoptó. Como es sabido, está construyendo en Asturias un complejo petroquímico de pesadilla.

La empresa Du Pont de Nemours es un modelo de empresa contaminante y destructora. Según la Revista Integral, de septiembre de 1991, «En su propio país fue denunciada y multada a finales de los años 80 por sus vertidos de residuos tóxicos, por contaminar aguas subterráneas, suelo y atmósfera, por el contenido en plomo de su gasolina y por los peligrosos residuos de su fungicida Manzate, que llegaban a los productos alimentarios. Además, tiene pendiente, desde 1989, una factura de $500 \mathrm{mi}-$ llones de dólares, a pagar fraccionadamente durante las próximas décadas, destinada a la limpieza y descontaminación de catorce lugares de Delaware, Nueva Jersey, Nueva York, Texas, Virginia y Washington, contaminados por ella".

Cualquier persona daría dinero por mantener a una empresa así alejada de su entorno. Pues bien, no lo entiende de esa manera nuestra 
Administración $y$, ni corta ni perezosa, le pide, le ruega y le subvenciona para que se instale en España, uno de los pocos países, que se sepa, que ampara constitucionalmente el llamado derecho al medio ambiente.

Para muestra sirve un botón. Pero España, en materia de ecología marcha delante de casi todos los países eurooccidentales contaminantes. Los agresores del ambiente o no son castigados, o la sanción es tan ridícula que puede ser incluida entre los gastos generales de cualquier empresa de tres al cuarto. España, se ha escrito, es "el paraíso de los plaguicidas". Pero que sean nuestros propios gobernantes los que fomenten y apoyen económicamente a potenciales amenazas es casi inconcebible.

IV. La precedente introducción viene a propósito de un asunto, absolutamente abstracto para la mayoría de nuestros conciudadanos, cual es el del carácter normativo y vinculante de nuestra Constitución, de todos y cada uno de sus preceptos. No sólo la letra del texto constitucional, en su artículo 9.1, sino la generalidad de la doctrina encabezada por el eminente jurista García de Enterría y el Tribunal Constitucional, vienen insistiendo en la aplicabilidad directa de la norma suprema, de toda ella.

Qué duda cabe, que buena parte de los preceptos constitucionales están vigentes en el pleno sentido de la palabra. Otros, empero, o se han ignorado, o son pura y simplemente música celestial o, en fin, son, en las circunstancias reales existentes, de aplicación imposible.

Consideremos, por ejemplo, algunos de los "derechos" pertenecientes a esa categoria, que algunos llaman "de prestación», derechos en función de la igualdad, claim rights, derechos "positivos", "económico-sociales", derechos humanos o fundamentales "de la segunda generación" -para diferenciarlos de los civiles y/o políticos-. En nuestra Constitución, aparecen dispersos, diseminados, por todo el Título l. Sin pretender ser exhaustivo, es posible, no obstante, localizar y destacar algunos de ellos:

- El derecho a recibir información y el de acceso a los medios de comunicación social dependientes del Estado (arts. 20.1d) y 20.3).

- El derecho a la educación (art. 27.1 y 4), cuya dimensión uprestacional" (término horrible donde los haya) se ha reconocido respecto de, y sólo de, la educación básica.

- El derecho al trabajo (arts. 35.1 y 25.2), que curiosamente constituye un derecho y un deber para el ciudadano normal, mientras 
que es un derecho - textualmente protegido con el recurso de amparo, por mucho que el Tribunal Constitucional no lo reconozca como tal一, pero no un deber, para el que ha delinquido. Una forma, sin duda, de promover -al revés, claro- "las condiciones para que la igualdad sea real y efectiva".

- El derecho a la protección de la salud (art. 43.1, en relación con el 51.1).

- El derecho a la cultura (art. 44.1).

- El derecho a disfrutar de un medio ambiente adecuado para el desarrollo de la persona, así como jel deber de conservarlo! (art. 45.1).

- El derecho a disfrutar de una vivienda digna y adecuada (art.47) (éste reservado sólo a los españoles).

Si exceptuamos el derecho a la educación básica -único que hasta el presente parece exigible y garantizado-, y el derecho a recibir información (que el Tribunal Constitucional, en una interpretación que no comparto, ha calificado de derecho de libertad frente al poder - lo que le convierte en un derecho negativo-liberal-), el resto de los "derechos" antes mencionados equivale a una mera declaración de intenciones. Intenciones frustradas $a b$ origine en bastantes casos. En otros, el desarrollo de la política económica y social ha producido los efectos contrarios, de tal forma que, por ejemplo, no sólo los presuntos derechos se han revelado irrealizables, sino que el deficiente funcionamiento de la Administración y la falta de medios han dado como resultado paro, en lugar de trabajo, intoxicaciones - no ya sólo nuevas enfermedades-, en lugar de salud, muerte y polución, en lugar de conservación de la vida y del entorno, empobrecimiento del idioma y la cultura, en lugar de incremento de las luces.

El problema, en este contexto, no es exclusivamente jurídico. No se trata pura y simplemente de mostrar que los "derechos" económico-sociales, no son accionables, ni exigibles, ni justiciables, por mucho que constituyan obligaciones formales del Estado o poderes públicos o «determinaciones de los fines del Estado". No es que no sean derechos al modo de los derechos públicos subjetivos. La calificación como derechos de algunas necesidades humanas no sirve de mucha ayuda ceteris partibus. Del mismo modo que en otro tiempo se hablaba de las "condiciones objetivas de la revolución" y se condenaba en buena lógica el voluntarismo, porque conducía a decisiones erróneas que costaban muchas vidas, la mayoría de los derechos económicos-sociales exige para su satisfacción la creación 
de una situación objetiva de prosperidad que de momento no existe. Pero no es eso todo, se dan supuestos en los que ni la riqueza puede auxiliarnos. Afirmar que se puede garantizar hoy en día, no ya en España, sino en Alemania o en Suecia, un medio ambiente saludable es de una ignorancia supina de la realidad $y$, además, significa carecer del mínimo sentido de la ubicación en el mundo actual. Sólo una acción internacional global, conjunta e integrada, podría empezar a contrarrestar el gravísimo y profundo deterioro del planeta. Un esfuerzo de ese tipo, principio de la creación de las condiciones objetivas para la existencia de un ecosistema limpio, no se ha iniciado aún.

Problema distinto, pero en íntima relación con la adopción, y probable exigibilidad, de un conjunto de "derechos" económico-sociales, es en España la tradicional, persistente y deseperante ineficacia de los servicios encomendados al Estado y otros entes públicos. No estoy pensando en la sobrecarga de funciones atribuidas a los modernos Estados del bienestar -hoy en crisis por las políticas de desregulación introducidas por Reagan y Thatcher-, ni tampoco en el posible conflicto entre los derechos liberales y los derechos positivos, sino los problemas de funcionamiento implícitos en una intervención creciente del Estado. Por paradójico que resulte, si nos atenemos a las corrientes dominantes en materia de gobierno, la consagración del Estado social ha llegado tarde. Asistimos así a una práctica política de un Gobierno que contradice los pronunciamiento literales de nuestra Constitución: los servicios esenciales para la sociedad no funcionan (justicia, sanidad, transportes, correos, etc.) y, además, se está alentando como consecuencia de ello la duplicación de esos servicios en el ámbito de la sociedad civil. De un partido en el Gobierno que legaliza la privatización de los servicios de policía y de una oposión que propone idéntica conducta respecto del régimen carcelario, no puede esperarse sino una política de desregulación que va a convertir al Estado y a la mayor parte de su funcionariado en un parásito de la sociedad civil.

\section{IMPORTANCIA DEL PROBLEMA}

La importancia del problema no necesita ser subrayada porque es el punto de cruce de muchos otros, todos centrales para nuestra Constitución, y varios ya aludidos en la introducción: el Estado social y democrático de derecho; el carácter normativo-directo de la Constitución, la igualdad formal y la material, y hasta el papel de los jueces, sin olvidar, en fin, la relación de España con la Comunidad Europea (y, con ello, el problema de si la Constitución va a ser suprema o no). Es claro que, hasta el momento, 
Bruselas no muestra una preocupación social como la que hay en el texto de nuestra Constitución.

\section{ALGUNAS ACLARACIONES}

A) Al valorar esta parte de la Constitución no discutimos que deba existir una sociedad civil viva y que deba haber por parte de la misma una solidaridad social, y, por parte de los poderes públicos, una asistencia en favor de los más débiles, en general. Por el contrario, se parte de esa base. Pero se discute aquí, en cambio, cómo se pueden consagrar constitucionaimente tales obligaciones y hacerlas cumplir.

Parece que tal solidaridad y asistencia deben existir, aunque no está claro si siempre corresponden en primer lugar a la sociedad civil (por ejemplo, el cuidado de los niños y ancianos) o a los poderes públicos (por ejemplo, el cuidado del medio ambiente). Si una sociedad es insolidaria no habrá Constitución que la haga solidaria, pues, como decía BAGEHOT, "no se puede hacer buenos a los hombres por ley del Parlamento". Las proposiciones que aparecen aquí no pretenden ofrecer garantías totales porque, sencillamente, tales cosas no existen en el campo del Derecho Constitucional. A título personal, los autores de estas líneas preferirian magnas cartas breves, democrático-liberales, que no se ocupen mucho de estos asuntos; no porque se defienda aquí la insolidaridad, sino porque se presupone una sociedad con una cierta salud moral que, por tanto, no será indiferente ante la suerte de los desposeídos, niños, ancianos, marginados, etc.

Ahora bien, es importante tener presente en todo momento que reformar la Constitución no es hacer ejercicios académicos ni exponer teorías propias. El realismo nos recuerda que:

1) Como norma, es mejor no reformar demasiado las Constituciones.

2) Pero si una Constitución, o parte de ella, fuera sistemáticamente incumplida, y se persistiera en no reformarla, los efectos de ello podrian ser perjudiciales para la propia Constitución por razones obvias. Pues bien, ese parece ser el caso de los derechos sociales y principios rectores a la altura de 1992. 
3) Parafraseando a BURKE, diremos que reformar una Constitución no es garrapatear arbitrariamente en una página en blanco, sino moverse entre los datos reales de lo que hay y lo que razonablemente se puede esperar de la reforma.

4) Los derechos sociales y sobre todo los principios rectores no se cumplen y existen escasas expectativas de que las líneas políticas esenciales cambien radicalmente a corto plazo, lo que aconsejaría suprimirlos en su mayoría para evitar el mal efecto de su constante violación. Además, no forman parte del núcleo irreductible de una magna carta.

5) Pero la sociedad española de hoy no es solidaria, lo que sugiere que no es éste el momento para eliminar de la Constitución toda referencia a la asistencia social, pues es de temer que el efecto de tal supresión fuera como echar gasolina al fuego o terminar de matar al moribundo.

6) Por otra parte, el texto del que partimos es muy social en su tenor literal, lo cual, combinado con el criterio de reformar sólo lo necesario, aconseja evitar los giros de 180 grados.

7) En cuanto a la eficacia práctica de estas disposiciones constitucionales recordaremos que, si bien no es posible para la Constitución obligar a un Gobierno a cumplir todos sus deberes asistenciales ni a dar aplicabilidad inmediata a la solidaridad ni a la mayor parte de los derechos sociales, sí puede ser posible judicializar aspectos particulares, o impedir algunas políticas anti-sociales.

8) Por tanto, la línea seguida aquí consiste en evitar los giros radicales, mantener de alguna forma la referencia a la obligación social de los poderes públicos (la de la sociedad se da por supuesta), retirar de la Constitución los derechos sociales y principios rectores que sean manifiestamente ociosos, redundantes o imposibles de cumplir, y traducir a un lenguaje jurídico concreto los que queden.

B) El primer problema está en que no sabemos cuál va a ser la política que siga la Comunidad Europea en estas materias, pues su preocupación social hasta ahora no es muy grande. Para el caso de que Bruselas si- 
guiera sin mostrar mucha sensibilidad social, conviene preguntarnos: ¿va a reservarse España alguna parcela de soberanía —en este caso, en el campo de la política social- que le permita garantizar unos mínimos sociales, cualquiera que sea la política seguida por Europa? Las fórmulas para ello serían variadas, pero en el fondo similares:

- relación federal como la que Quebec pretende, en otro campo, con su "sociedad distinta";

- reservas como las furmuladas en Maastricht, también en otros campos, por el Reino Unido e Irlanda;

- «no centralización» (como la llama el profesor Elazar): no entregrar a Bruselas determinadas materias;

- reservas como las de Escocia frente al Parlamento de Westminster consagradas en los Tratados de Unión de 1706 y 1707;

— alguna solución similar al antiguo "pase foral» vasco y navarro.

Si España perdiera toda autonomía en este campo la solución sería bien fácil: añadir un número más al artículo 53 de la Constitución, diciendo:

"En todas las materias sociales se estará a lo que dispongan las políticas sociales de la Comunidad Europea en cada momento" (sería el nuevo 53.4).

Aquí se da por supuesto que, por las vías que sea, Madrid retendrá un grado sustancial de capacidad para formular políticas sociales autonómicamente.

C) Llegados a este punto puede ser procedente recordar el ejemplo del Canadá: un país que siempre ha tenido una Constitución liberal, la cual no le impidió desarrollar una tradición de asistencia social, que comparte con el Reino Unido. El Estado social canadiense se concreta, sobre todo, en la asistencia pública en materias de sanidad, educación y cultura, vivienda, desempleo $y$ pensiones, y ayuda familiar e infantil. Todo ello forma una red asistencial envidiada, con razón, por otros países. Con motivo de los actuales debates sobre la reforma constitucional está poniéndose de relieve el temor de muchos canadienses a que esa dimensión asis- 
tencial corra ahora peligro, a causa de los siguientes factores: el acuerdo de libre comercio con los Estados Unidos y sus consecuencias, las modernas políticas económicas $y$, por unas razones específicamente canadienses, que ahora no nos ocupan, las actuales tendencias descentralizadoras.

En esta situación, los defensores de la "opción social" parecen no saber exactamente qué partido tomar:

- promulgar una carta de derechos sociales (social charter) no exigible ante los tribunales pero protegida por algún nuevo órgano, probablemente de naturaleza consultiva;

- garantizar unos mínimos standards sociales por medio de una interpretación social de los derechos liberales ya reconocidos en la vigente Charter of Rights and Freedoms;

- promulgar una carta de derechos sociales inmediatamente exigibles ante los tribunales;

- otras posibilidades menos interesantes para nosotros ahora.

(Sobre Canadá, ver Will KYMLICKA y Wayner J. NORMAN: "The Social Charter Debate: Should Social Justice be Constitucionalized?", Analysis, 2, II-1992).

El ejemplo canadiense ofrece varias lecciones: la primera, que a la hora de construir una red asistencial fue más importante la cultura política (de asistencia social) que el texto constitucional (clásico y liberal); la segunda, que los problemas que ahora afectan a los derechos sociales españoles no son una particularidad de este país; la tercera, los riesgos que conllevan las modernas uniones supra-nacionales, y la cuarta, la dificultad para garantizar constitucionalmente unos derechos sociales cuando las políticas económicas de moda son contrarias. Y, así como la ausencia de dimensión social en la Constitución canadiense no impidió el desarrollo de un Estado asistencial, uno se pregunta: si las cosas toman el rumbo contrario, ¿podría ahora la pretendida constitucionalización de tales derechos sociales impedir el naufragio?

D) El aspecto clave parece ser, en última instancia, no el matiz político o económico de los derechos sociales, ni su carácter positivo o de prestación, pues la frontera entre unos y otros derechos no es clara, como se advierte en la Introducción. El problema clave parece ser cómo protegerlos constitucionalmente de una manera efectiva, y para ello, en primer 
lugar, cómo configurarlos de tal manera que puedan ser alegados ante un juez en el curso de un pleito. Es evidente que las pretensiones, subvenciones y prestaciones sociales de naturaleza económica o política no son tan fáciles de traducir a lenguaje jurídico como los clásicos derechos negativos. Pero también es cierto que determinados problemas políticos han sido judicializados con razonable éxito (ejemplo: conflictos federales).

La experiencia también muestra que los derechos sociales, cuando son concretados en una ley y dotados de un capítulo presupuestario y de unos procedimentos, pueden ser judicializados. Pero eso es como reconocer que dependen de la ley y del reglamento, no de la Constitución. De ahí se podría deducir que si, al fin y al cabo, van a depender del legislador infra-constitucional, su constitucionalización sería superflua. De ahi el criterio, seguido en esta propuesta de reforma, de no incluir en la Constitución derechos que pueden cumplirse siempre que los reconozcan leyes, reglamentos, y órganos administrativos prestadores. Además, la lista de tales derechos sería interminable.

Si concluimos que su naturaleza es jurídica, hay que hacerlos justiciables y dotarlos de una eficaz protección jurídica. Sí, por el contrario, por su naturaleza, fueran metas políticas, habría que dotarlos de una protección política. Es claro que ninguna de ambas cosas parece fácil. Si son aspiraciones o valores sociales (y algunos lo son, o mucho lo parecen), la experiencia española de estos años ha mostrado que es muy difícil positivar valores y hacerlos entrar en el mundo de lo jurídico.

\section{STATU QUO}

La posición jurídico-constitucional de los derechos sociales y principios rectores de la política social y económica es bien conocida. La magna Carta española es, en su totalidad, normativa-directa, de acuerdo con su artículo 9.1 y con numerosa jurisprudencia constitucional y con no menos numerosa doctrina. Como se dijo en la Introducción, la mayor parte de estos derechos están en los arts. 30 a 52 de la Constitución, aunque otros están en otras partes, incluyendo la lista de los arts. 14 a 29, protegida por recurso de amparo. El art. 53, al pronunciarse sobre la protección que otorga a los derechos, viene a decirnos cuán en serio se los toma y, en cierto modo, viene a supender la vigencia del 9.1 en estas materias. La jurisprudencia constitucional, que es abundante, no les resulta muy favorable, pues el Alto Tribunal, al fin y al cabo, nunca los ha considerado como derechos ex constitutione sino ex lege, llegando a retirar el amparo incluso a 
alguno de los derechos comprendidos entre los arts. 14 al 29 . Simplificando, podría seguramente decirse que el derecho social que la jurisprudencia constitucional parece considerar más sustancial es la clásica igualdad, liberal-democrática, del art. 14. A estos derechos y principios rectores los hace depender de la ley, de la existencia de recursos económicos de una Administración subvencionadora 0 , en fin, de que puedan ser reconducidos al citado derecho "fuerte», "serio», del art. 14. (Para no alargar demasiado esta recapitulación, puede verse: PEREIRA MENAUT: "Against Positive Rights", en Valparaiso University Law Review, 22, 1988, 359-383; COELLOPEREIRA: "Normativa constitucional, igualdad y derechos sociales", en El Principio de Igualdad en la Constitución española. Madrid, 1991, vol. I, 941957, y PEREIRA-TIRAPU: "Observaciones sobre la jurisprudencia constitucional en materia de igualdad", en El Principio de Igualdad..., I, 959-974).

\section{OPCIONES}

Antes de pronunciarnos sobre lo que se puede proponer, conviene pensar en lo que puede razonablemente esperarse de la política social española en el próximo decenio. Con la experiencia de casi catorce años de funcionamiento de la Constitución, y con unas perspectivas todavía más claramente anti-sociales, no parece recomendable embarcarse en reformas que tengan escasas posibilidades de ser cumplidas. Habria que evitar mandatos al legislador que nunca podrán ser exigidos, proclamaciones de derechos inviables o que vengan a traducirse sólo en refuerzos del poder ejecutivo, enunciados de valores que después no podrán ser positivados... El mencionado ejemplo canadiense ilumina varias cosas: entre otras, la perplejidad ante la solución que deba adoptarse.

Las opciones que se abren ante nosotros pueden ser resumidas como sigue:

A) Retirar de la Constitución todos los derechos sociales o positivos y principios rectores, incluyendo también el art. 9.2 y el "estado social» del art. 1.1. Parece una solución realista, sobre todo si las políticas económicas europeas no dejan mucho margen de maniobra. Pero tiene el inconveniente de ser demasiado radical, incluso desmoralizadora. En la práctica, abstenerse de añadir una carta social a una Constitución democrático-liberal que ya funciona — una buena posibilidad para los canadienses y otros-. es muy distinto de borrar toda disposición o mandato asistencial de una Constitución que tiene un compromiso social formal. No parece que siem- 
pre haya que elevar a nivel de ley todo lo que exista a nivel de calle. No es lo mismo tolerar de facto un cierto fraude fiscal que fomentarlo $y$, además, borrar de la legislación todo rastro de obligación de contribuir a las cargas sociales.

B) El realismo aconseja también no constitucionalizar la plena justiciabilidad de los arts. 30 a 52 (más 9.2, 25.2, 27.9, etc.), por lo menos rebus sic stantibus. Incluso aunque la situación económica fuera favorable, esta solución tendría diversos inconvenientes - como el activismo judicial excesivo-, en los que no nos detendremos ahora dado lo improbable que parece. (Esta opción, como las siguientes, presupone que España retendrá alguna autonomia frente a la Comunidad Europea.)

C) Admitiendo la naturaleza más política (o social, o económica) que jurídica de los llamados derechos positivos, otra opción consistiría en explorar la posibilidad de dar a los derechos sociales una protección política. De la misma manera que a nadie se le ocurre judicializar las diferencias políticas entre el legislativo y el ejecutivo, que tienen su propia sede y procedimientos (Parlamentos, controles, mociones de censura, $y$, en última instancia, las elecciones), se podría intentar buscar garantías políticas y sociales para estas realidades de naturaleza política y social. El problema reside en cómo concretar las tales garantías. Desgraciadamente, la experiencia del constitucionalismo comparado no ofrece gran ayuda en este caso. ¿A qué organismo se podría encargar ese control político? Hoy por hoy, no a las Cortes, ni al Defensor del Pueblo, ni a ningún órgano partidario. Los canadienses hablan, en esta línea, de una especie de consejo de expertos cuya tarea consistiría en emitir informes sobre las políticas sociales y sugerir líneas de actuación. Pero si no tiene competencias concretas, corre el riesgo de servir de poco. En los países anglosajones suele haber cuerpos públicos o semi-públicos independientes que emiten informes que luego son tenidos en cuenta, pero en España falta esa tradición de auctoritas sin potestas, así que parece que nos vemos forzados a elegir entre órganos con poderes efectivos o nada.

La solución menos mala sería, quizá, que ese órgano independiente tuviera funciones consultivas y de asesoramiento sin iniciativa legislativa, pero pudiendo vetar las leyes o políticas declaradamente anti-sociales.

D) Como una solución ideal no existe, aquí se propone la combinación siguiente: 
1) Suprimir de la Constitución los artículos de contenido social, dejando únicamente la cláusula del estado social del art. 1.1, de por sí suficiente.

2) Crear un órgano independiente con las funciones mencionadas $y$ competencia para vetar, en casos de políticas manifiestamente insolidarias o anti-sociales. El veto sería superable por una mayoría parlamentaria cualificada.

3) Dotar a la cláusula social, antes citada, de una eficacia aminorada pero concreta. Por ejemplo, en nombre de esa cláusula no se podría exigir a un Gobierno sin recursos que adoptara nuevas líneas de asistencia social, pero sí que no cortara injustificada o bruscamente las existentes. Para esto cabría el control de constitucionalidad de leyes y normas de rango inferior a la ley, y el recurso de amparo para los afectados por las agresiones positivas e injustificadas a alguna de las políticas sociales existentes (no por la inexistencia de un programa asistencial en un momento de vacas flacas). Dentro de lo malo, no es lo mismo dejar fuera de nuestra casa a un mendigo en una noche invernal, que primero admitirlo y luego expulsarlo. Así, no serían amparables el derecho a la información, el derecho a un puesto de trabajo ni el derecho a una vivienda digna, pero sí el derecho a que el Estado no subvencione a Du Pont de Nemours ni corte bruscamente por completo la ayuda familiar o el subsidio de desempleo mientras que no recorta otros gastos. Si la cesación de esas ayudas se hiciera por medio de una ley o reglamento podría ser objeto de control de inconstitucionalidad; si se hiciera por medio de simples actos administrativos podría ser objeto de recurso de amparo por los afectados o de veto por el órgano mencionado.

4) Proteger, declarándolos expresamente justiciables, en el art. 53, todos aquellos derechos económicos y sociales que por su naturaleza lo admitan -obviamente, no muchos-, y retirar los demás. Deberian también ser retirados los que, aun siendo justiciables, puedan ser reconducidos a algún derecho "fuerte" ya existente, en particular a la igualdad - por ejemplo, la igualdad en el acceso a una prestación o subsidio-, como ocurre con varios de ellos.

Recordaremos que los derechos sociales y principios rectores no forman una categoría unitaria, lo que repercute en la diversa dificultad de su judicialización. Más en concreto, y sabiendo el limitado alcance que ten- 
dría en la practica una reforma, se ofrecen aquí la siguientes modestas soluciones.

\section{NUEVO ARTICULADO}

\section{TITTULO PRELIMINAR:}

-Art. 1.0-4:

"Los poderes públicos cuidarán del medio ambiente, la salud, la educación, el empleo, las pensiones y demás obligaciones de un Estado social. Ayudarán a los ciudadanos necesitados y débiles".

-Art. 9.2: debería ser suprimido.

CAPITULO II, SECCIÓN I:

-Art. 10.2, b) (derecho a la producción literaria, etc.): suprimido.

-Art. 20.1, d) (derecho a recibir libremente información veraz): suprimido.

-Art. 25.2 (derecho de los presos al trabajo): suprimido.

-Art. 27.5 (derecho a la educación en general): suprimido.

-Art. 27.9 (derecho ex lege de los centros docentes a subvenciones): suprimido; su rango constitucional queda en entredicho con esa remisión de la propia Constitución a la ley. 
CAPÍTULO II, SECCIÓN II:

-Art. 30 (derecho a defender a España; objeción de conciencia): suprimir los números 1 y 4 .

-Art. 31 (impuestos): suprimirlo, excepto la referencia genérica a la justicia, progresividad y legalidad de los tributos. Añadir: "no habrá impuesto sin consentimienton. Protección contra impuestos injustos o anti-constitucionales: contra leyes y reglamentos, recurso de inconstitucionalidad; amparo, para los particulares, una vez agotada la vía ordinaria. (N. B.: se parte de la base de la función de super-supremo que está ya ejerciendo el Tribunal Constitucional en ciertos respectos).

-Art. 32 (matrimonio, divorcio): suprimido.

-Art. 33 (propiedad): suprimir el numero 3 por superfluo y por remitirse a las leyes; proteger los números 1 y 2 con recurso de amparo.

-Art. 34 (fundación): suprimido por superfluo y porque la propia Constitución se remite a la ley. Recordemos el antedicho criterio: la Constitución no es para recoger enumeraciones de derechos legales.

-Art. 35 (derecho al trabajo): suprimido por razones obvias.

-Art. 36 (colegios profesionales): suprimirlo por superfluo y por la remisión constitucional a la ley.

-Art. 37.1 (negociación laboral): suprimido.

-Art. 38 (economía de mercado): suprimido.

CAPÍTULO III:

En primer lugar, se debería suprimir el epígrafe que dice "Capítulo II", de modo que continuase el articulado sin interrupción, renumeran- 
do los capítulos IV y V (ahora III y IV). El capítulo II resultaría agrandado y lo que ahora es el III dejaría de existir independientemente.

Motivos: el primero, que lo que queda de los arts. 39 a 52 puede ser incorporado sin problemas a la sección segunda del capítulo II, y el segundo, que la ineficacia de estos artículos ha quedado probada en estos catorce años. En cuanto a cláusulas generales o interpretativas: de las obligaciones sociales del Estado y del nuevo art. 40 pueden deducirse criterios generales (no concretos, que no hacen falta) que orienten la política social y económica.

Por lo que se refiere al articulado:

-Art. 39: dejar la cláusula genérica del numero 1 y suprimir los tres números restantes: el 2 y el 3 por ser reconducibles a la igualdad, y el 4 por ser una cuestión de tratados internacionales, superflua en esta sede.

-Art. 40 (política de pleno empleo, etc.): sustituirlo por la siguiente redacción:

"Los poderes públicos promoverán las condiciones favorables para el desarrollo social y económico y para una distribución de la renta regional y personal más equitativa. En ningún caso llevarán a cabo políticas manifiestamente anti-sociales, salvo en situaciones de emergencia, durante cortos períodos, $y$ con razones suficientes, que apreciará el órgano a que se refiere el art. 53.3" (en su nueva redacción).

-Art. 41 (seguridad social): suprimido.

-Art. 42 (emigrantes): suprimido.

-Art. 43 (salud, deporte, ocio): suprimido.

-Art. 44 (acceso a la cultura, ciencia, investigación): suprimido.

-Art. 45 (medio ambiente): merece un comentario aparte. En esta materia, España, que va atrasada, estará cada día más obligada por sus compromisos internacionales y por la Comunidad Europea. Por otra parte, el medio ambiente es una materia muy am- 
plia, divisible en varias parcelas con tratamientos separados, unas susceptibles de protección judicial (siempre que los afectados y los daños sean identificables; recuérdese el caso romano de la Taberna casiaria y otras imposibles de judicializar por estar fuera del alcance de la propia Constitución española (como el agujero de ozono). Los vertidos insalubres no necesitan constitucionalizarse por estar contemplados por la legislación ordinaria.

En la nueva redacción que aquí se propone no se considera protegible un derecho abstracto al medio ambiente, que es dudoso que exista, sino un derecho, concreto y judicializable, frente a acciones contra el medio ambiente, positivas e identificables, del Estado o de particulares:

"Todos tienen el derecho a disfrutar de un medio ambiente sano. Ni el Estado ni los particulares tomarán decisiones manifiestamente dañosas para el medio ambiente, contra las cuales cabrán los recursos legales ordinarios o bien los de inconstitucionalidad o amparo, si proceden.

-Art. 46 (patrimonio histórico): suprimido.

-Art. 47 (vivienda digna): suprimido.

-Art. 48 (juventud): suprimido.

-Art. 49 (minusválidos): suprimido.

-Art. 50 (tercera edad): suprimido.

-Art. 51 (consumidores): suprimido.

-Art. 52 lorganizaciones profesionales de defensa de los intereses económicos): suprimido.

CAPÍTULO III (antes IV):

-Art. 53.2: nueva redacción: 
"Cualquier ciudadano podrá recabar la tutela de las libertades y derechos reconocidos en el artículo 14 y el Capítulo segundo...".

-Art. 53.3: nueva redacción:

El nuevo órgano consultivo que sea creado (evitemos pronunciarnos sobre el nombre) "velara porque el Estado lleve a cabo sus obligaciones sociales. Recibirá quejas, podrá sumir la defensa de los perjudicados y evacuará dictámenes preceptivos pero no vinculantes. Vetará las normas y actos manifiestamente anti-sociales y contrarios a los artículos 1.1 y 40 . Para levantar su veto se necesitará una mayoría de dos tercios en ambas Cámaras.

Estará legitimado para interponer recursos tanto ordinarios como de inconstitucionalidad y de amparo, si procedieren". 